\title{
Muscle-eye-brain Disease and Drug-resistant Seizures: Unravelling the Phenotypic Heterogeneity of Congenital Muscular Dystrophies
}

Deepak Menon, Joseph Samuel, Sai Satish, Ramshekhar Menon, Bejoy Thomas and Ashalatha Radhakrishnan

Department of Neurology, Sree Chitra Tirunal Institute of Medical Sciences and Technology, Trivandrum, Kerala, India

"Corresponding author: Dr Ashalatha Radhakrishnan, R. Madhavan Nayar Center for Comprehensive Epilepsy Care, Department of Neurology, Sree Chitra Tirunal Institute of Medical Sciences and Technology, Trivandrum, Kerala, India, Tel: 91- 471 - 2524282; E-mail: drashalatha@sctimst.ac.in

Received date: June 10, 2016; Accepted date: June 20, 2016; Published date: June 25, 2016

Copyright: (C) 2016 Menon D, et al. This is an open-access article distributed under the terms of the Creative Commons Attribution License, which permits unrestricted use, distribution, and reproduction in any medium, provided the original author and source are credited.

\begin{abstract}
Muscle-Eye-Brain Disease (MEB) constitutes part of a spectrum of closely overlapping Congenital Muscular Dystrophies (CMD) and neuronal migration disorders. Here, we present a child with MEB presenting with refractory epilepsy, a rare disease and all the more, a rare presenting manifestation. We hereby highlight the rarity of the syndrome per say, its presentation as refractory seizures to an Epileptologist and the radiological characteristics which help diagnosing MEB accurately obviating the need for an invasive procedure like muscle biopsy and molecular genetic studies in centres with limited infrastructure.
\end{abstract}

Keywords: Muscle-eye-brain disease; Refractory seizures; MRI

\section{Case Report}

A one year-eight month-old baby boy born of non-consanguineous parentage, with no antecedents, except for global developmental delay presented to our Comprehensive Epilepsy Care Program with refractory seizures since seven months of age. The seizures were initially characterised by head deviation to right followed by right hemiclonic seizures during sleep occurring 1-2 per month lasting 2-3 min each and had gradually ran up over time to 2-3 per week. Since the age of one year, parents also noticed events with eye deviation to one side followed by facial twitches lasting 10-15 sec. There was no relevant family history, neither were then any other systemic symptoms as reported by the family whose only concern was seizures. Child had been on sodium valproate with little improvement in seizures.

His examination revealed microcephaly, poor visual regard, concomitant strabismus, generalised hypotonia and absent deep tendon reflexes. There were no neurocutaneous markers or remarkable musculoskeletal or systemic anomalies. His overall developmental quotient was four months. His video-EEG revealed diffuse slowing of background in awake state $(3-4 \mathrm{~Hz}$, polymorphic medium amplitude, delta), poorly formed scarce sleep structures, with multifocal interictal epileptiform discharges predominantly over both fronto-temporal regions with sleep activation of generalised discharges and generalised paroxysmal fast activity suggesting an epileptic encephalopathy. One symmetric tonic seizure was recorded characterised by abduction of both hands with stiffening and uprolling of eyes, of diffuse electrical onset with an electrodecremental pattern as ictal correlate.

MRI T2 sagittal sequences showed pontine hypoplasia, fusion of superior and inferior colliculi and dilated fourth ventricle (Figure 1); T2 axial sequences revealed bilateral lateral cerebellar cysts (Figure 1), diffuse hypomyelination predominantly affecting the anterior temporal subcortical regions and frontal cobble stone lissencephaly (Figure 2). In addition, there was posterior buckling of the sclera suggesting a posterior staphyloma (Figure 2).

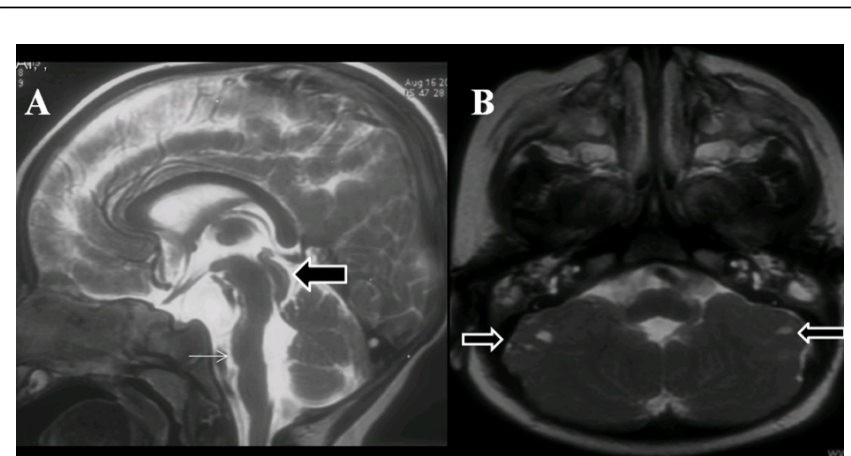

Figure 1: (A) MRI sagittal T2 images showing pontine hypoplasia (white arrow), enlarged fourth ventricle, and fusion of superior and inferior colliculi (black arrow) (B) Coronal T2 image shows lateral cerebellar cysts (black arrows).

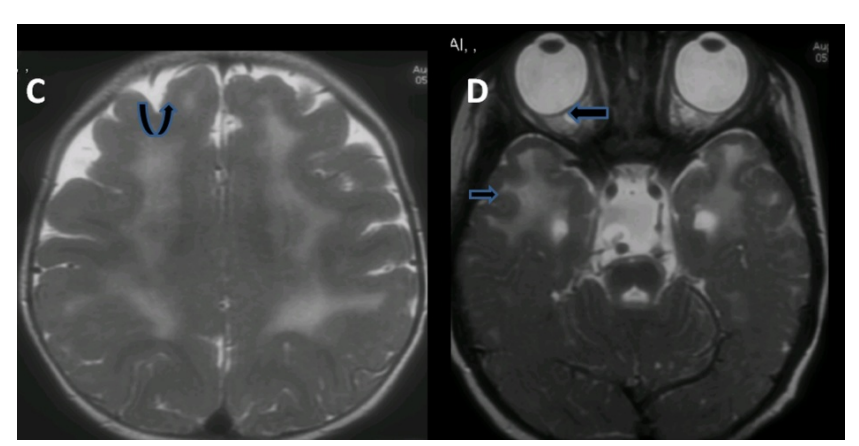

Figure 2: (C) Axial T2 images showing hypomyelination in cerebral white matter, more pronounced anteriorly over temporal lobes and frontal cobble stone lissencephaly (curved arrow (D). Axial T2 images showing posterior staphyloma of orbit (large arrow) and anterior temporal hypomyelination (small arrow). 
There was elevated creatine kinase levels $8220 \mu / \mathrm{L}$ (normal 21-22 $\mu / \mathrm{L}$ ), bilaterally prolonged VEPs and EMG from rectus femoris showed myopathic pattern. Child was diagnosed as MEB and has been initiated on valproate and lamotrigine combination and rehabilitative measures.

\section{Discussion}

MEB together with Walker-Warburg syndrome and Fukuyama congenital muscular dystrophy, form a very rare heterogeneous group of disorders and forms the borderland between congenital muscular dystrophies and neuronal migration disorders. Walker-Warburg syndrome is the most severe form and affected children usually do not survive beyond three years of age and have severe generalized weakness and hypotonia in infancy, has severe visual impairment secondary to several ocular malformations and later develop respiratory insufficiency. Epilepsy can be a feature in them, although not very common and seldom refractory enough to seek attention of an epileptologist.

The sub-classification of congenital muscular dystrophies is based on the location of the defective proteins and the genetic mutations involved and thus is classified as those due to mutations of structural proteins of basal lamina, impaired alpha-dystroglycan glycosylation (dystroglycanopathies) and selenoprotein 1 mutations. Among the group of dystroglycanopathies, there is striking heterogeneity with the clinical spectrum ranging from most severe muscular dystrophydystroglycanopathy with brain and eye anomalies (MDDG type A), previously designated Walker-Warburg syndrome (WWS) or muscleeye-brain disease (MEB), the intermediate variety with or without mental retardation (MDDG type B) which includes the Fukuyama's congenital muscular dystrophy and the less severe form which presents as limb girdle muscular dystrophy (MDDG type C). At present, mutations in no less than 13 genes involved in the glycosylation pathway (POMT1, POMT2, POMGnT1, FKRP, Fukutin, LARGE, ISPD, GTDC2, B3GALNT2, B3GNT1, TMEM5, GMPPB, SGK196) have been identified. Various studies that sought to establish a genotype-phenotype correlation has failed to prove such an association and it thus follows that the clinical phenotype may not predict the associated mutation.

Though the spectrum of CMD is wide with one entity almost imperceptibly merging with the other in terms of its clinical involvement, the traditional classification among the dystroglycanopathies, namely WWS, MEB and FCMD still holds true especially in the resource-poor setting where genetic testing is beyond the reach of many. Here, the clinical and radiological assessment still goes a long way in differentiating between these closely overlapping phenotypes. Affected children present with delay or arrest of gross motor development with joint and/or spinal rigidity. Muscle weakness may improve, worsen, or stabilize in the short term; however, with time progressive weakness and joint contractures, spinal deformities, and respiratory compromise may affect quality of life and life span. MEB, initially described by Santavuori [1], consists of limb and axial hypotonia, global developmental delay, but eventually most can stand and some even ambulate with support. The extent of ocular involvement can vary from congenital glaucoma, optic disc pallor, retinal hypoplasia, posterior staphyloma and high myopia.

The hallmark MRI findings in dystroglycanopathies include multiple irregular small cotical gyri, cobblestone lissencephaly, fused midbrain colliculi, pontine and vermian hypoplasia, dilated fourth ventricle, lateral cerebellar cysts (which are frequently observed in POMGnT1, and FKRP mutations and have recently been described in POMT2 and LARGE patients) with or without cerebellar polymicrogyria, patchy hypomyelination and callosal hypogenesis variably $[2,4-6]$. MRI in Walker-Warburg syndrome shows much greater degree of abnormalities with complete absence of cerebral and cerebellar myelin, hydrocephalus and severe cobblestone lissencephaly $[2,3]$. Fukuyama congenital muscular dystrophy, seldom seen outside Japan, can be differentiated clinically from the two by calf hypertrophy, more severe contracture and myopathic facies. MRI may closely resemble that of MEB [2].

However, dominant presentation as refractory seizures is not a well characterised feature of CMD. Also, the diagnosis of CMD relies on clinical findings, brain and muscle imaging, muscle biopsy histology (dystrophic features without the hallmarks of the structural changes seen in the congenital myopathies), muscle and/or skin immunohistochemical staining, and molecular genetic testing. But here we emphasize the fact that careful analysis of clinical features, simple laboratory investigations and neuroimaging will help clinching the diagnosis and thereby may help avoid muscle biopsy and sophisticated genetic testing in countries with limited resources.

\section{References}

1. Santavuori P, Somer H, Sainio K, Rapola J, Kruus S, et al. (1989) Muscleeye-brain disease (MEB). Brain Dev 11: 147-153.

2. Barkovich AJ (1998) Neuroimaging manifestations and classification of congenital muscular dystrophies. AJNR 19: 1389-1396.

3. Cormand B, Pihko H, Bayes M, Valanne L, Santavouri P, et al. (2001) Clinical and genetic distinction between Walker-Warburg syndrome and muscle eye brain disease. Neurology 56: 1059-1069.

4. Valanne L, Pihko H, Katevuo K, Kartunnen P, Somer H, et al. (1994) MRI of Brain in muscle-eye-brain (MEB) disease. Neuroradiology 6: 473-476.

5. Bönnemann CG, Wang CH, Quijano-Roy S, Deconinck N, Bertini E (2014) Diagnostic approach to the congenital muscular dystrophies. Neuromuscul Disord 24: 289-311.

6. Godfrey C, Clement E, Mein R (2007) Refining genotype phenotype correlations in muscular dystrophies with defective glycosylation of dystroglycan. Brain 130: 2725-2735. 